\title{
Non-Gaussian Resistance Fluctuations in Disordered Materials
}

\author{
C. Pennetta ${ }^{1}$, E. Alfinito ${ }^{1}$, L. Reggiani ${ }^{1}$, S. Ruffo ${ }^{2}$ \\ ${ }^{1}$ INFM - National Nanotechnology Laboratory and Dipartimento di Ingegneria dell'Innovazione, \\ Università di Lecce, Italy, Via Arnesano, I-73100, Lecce, Italy, \\ 2 CSDC and Dipartimento di Energetica "Sergio Stecco", INFN and INFM, \\ Università di Firenze, Via S. Marta, 3, Firenze, 50139, Italy. *
}

\begin{abstract}
We study the distribution of resistance fluctuations of conducting thin films with different levels of internal disorder. The film is modeled as a resistor network in a steady state determined by the competition between two biased processes, breaking and recovery of the elementary resistors. The fluctuations of the film resistance are calculated by Monte Carlo simulations which are performed under different bias conditions, from the linear regime up to the threshold for electrical breakdown. Depending on the value of the external current, on the level of disorder and on the size of the system, the distribution of the resistance fluctuations can exhibit significant deviations from Gaussianity. As a general trend, a size dependent, non universal distribution is found for systems with low and intermediate disorder. However, for strongly disordered systems, close to the critical point of the conductor-insulator transition, the non-Gaussianity persists when the size is increased and the distribution of resistance fluctuations is well described by the universal Bramwell-Holdsworth-Pinton distribution.
\end{abstract}

Keywords:Non-Gaussian distributions, Disordered materials, Non-equilibrium steady states, Electrical breakdown

\section{INTRODUCTION}

In a macroscopic system, the fluctuations of a global quantity are generally expected to follow a Gaussian distribution around the average value. This expectation relies on the assumption, frequently true, that the system can be divided into statistically independent microscopic or mesoscopic elements. For such a system, the conditions of validity of the central-limit theorem are in fact satisfied and, consequently, Gaussian distributions of fluctuations are actually observed. On the other hand, when the correlations between the different elements of the system are significant, the centrallimit theorem is no longer valid and a non-Gaussian distribution of fluctuations is expected. Therefore, nonGaussian fluctuation distributions of global quantities are the signature of a correlated system. As correlations become important near the critical points of phase transitions, non-Gaussian distributions are usually observed near criticality ${ }^{1-7}$. In these conditions, the self-similarity of the system over all the scales, from a characteristic microscopic length up to the size of the system (which for a system of finite size sets the cut-off for the correlation length), has important implications on the fluctuation distribution ${ }^{1-4,8,5-7,9}$. Recently, new light has been shed on this subject by the remarkable discovery made by Bramwell, Holdsworth and Pinton (BHP) ${ }^{3}$ of a common behavior of the distribution of fluctuations of two quite different systems. Namely, the distribution of the fluc- tuations of the power consumption measured in confined turbulent-flow experiments and the distribution of magnetization fluctuations in a two-dimensional XY model in the spin-wave regime at low temperature ${ }^{3}$. Since these two systems appear to have very little in common, BHP advanced the reasonable suggestion that the origin of this common distribution should be attributed to scale invariance, the only property apparently shared by the two systems $^{3}$. This suggestion has been supported by the subsequent finding that many scale invariant systems, in both equilibrium and non-equilibrium steady states, display the same functional form for the distribution of fluctuations $s^{4,8,10,11,5}$. Concerning this point, it must be noted that the probability density function (PDF) of the distribution of fluctuations of a critical system can be considered as universal, in the sense that, when properly normalized, it depends on very few basic symmetries that define the universality class of the system ${ }^{8}$. Thus, the BHP distribution describes a universal behavior of the fluctuations of a critical system. In any case, it must be remarked that not all critical systems fluctuate according to this distribution ${ }^{5-7,11}$. On the other hand, far from criticality, the correlations between different elements of the systems can also be important. This is particularly true for systems in non-equilibrium steady-states, where non-Gaussian fluctuations are frequently observed ${ }^{1,2,7,12}$. Therefore the study of non-Gaussian fluctuations and the understanding of their link with other features of the sys-

${ }^{*}$ Corresponding authors e-mail: cecilia.pennetta@unile.it 
tem can provide new insights on some basic properties of complex systems ${ }^{1,2,4-7,11,9}$.

Here, we study the distribution of resistance fluctuations of conducting thin films of different size and with different levels of internal disorder. The resistance fluctuations are investigated under different bias conditions, from the linear response regime up to the threshold for electrical breakdown. This last phenomenon, which consists of an irreversible increase of the resistance, is thus associated with a conductor-insulator transition and it occurs in conducting materials stressed by high current densities $^{13-19}$. In our study we make use of the Stationary and Biased Resistor Network (SBRN) model ${ }^{20-22}$. This model provides a good description of many features associated with the electrical instability of composites materials $^{17,18}$ and with the electromigration damage of metal lines ${ }^{19,23}$, two important classes of breakdown phenomena. The film is modeled as a resistor network which reaches a steady state determined by the competition between two biased stochastic processes, breaking and recovery of the elementary resistors. The resistance and its fluctuations are then calculated by Monte Carlo simulations. Resistance fluctuations are found to deviate from Gaussianity near electrical breakdown. As a general trend, a non universal distribution of fluctuations is found for systems with low and intermediate disorder, where the deviations from Gaussianity vanish in the large size limit. However, for highly disordered systems, close to the critical point of the conductor-insulator transition, these deviations from Gaussianity persist when the size is increased and the distribution of resistance fluctuations is well described by the universal BHP distribution ${ }^{3,4}$.

\section{MODEL}

We describe a conducting film with granular structure as a two-dimensional resistor network. Precisely, we consider a square-lattice of $N \times N$ resistors, where $N$ determines the linear size of the network. This lattice lies on an insulating substrate at a given temperature $T_{0}$, which acts as a thermal bath. Each resistor can be in two different states ${ }^{13,24,19}$ : (i) regular, corresponding to a resistance $r_{n}=r_{0}\left[1+\alpha\left(T_{n}-T_{0}\right)\right]$, and (ii) broken, corresponding to an effectively "infinite" resistance, $r_{O P}=10^{9} r_{n}$ (resistors in this state will be called defects). In the previous expression of $r_{n}, \alpha$ is the temperature coefficient of the resistance and $T_{n}$ the local temperature. This latter is determined by Joule heating effects ${ }^{13,24,19}$ and thermal exchanges between neighbor resistors ${ }^{19}$ :

$$
T_{n}=T_{0}+A\left[r_{n} i_{n}^{2}+\left(3 / 4 N_{n e i g}\right) \sum_{m=1}^{N_{n e i g}}\left(r_{l} i_{l}^{2}-r_{n} i_{n}^{2}\right)\right]
$$

where, $i_{n}$ is the current flowing in the $\mathrm{n}$ th resistor and $N_{n e i g}$ the number of nearest neighbors over which the summation is performed. The parameter $A$ represents the thermal resistance of each resistor and sets the importance of Joule heating effects. By taking the above expression for $T_{n}$ we are assuming an instantaneous thermalization $^{19}$ of each resistor at the value $T_{n}$. The external bias consists of a constant current $I$ applied through perfectly conducting bars at the left and right sides of the network. In the initial state of the network (no external bias) all the resistors are identical: $r_{n} \equiv r_{0}$.

We assume that two competing biased processes act to determine the evolution of the network $\mathrm{k}^{20-22}$. These two processes consist of stochastic transitions between the two possible states of each resistor and they are taken to occur through thermal activation, with probabilities ${ }^{24}$ : $W_{D n}=\exp \left[-E_{D} / k_{B} T_{n}\right]$ and $W_{R n}=\exp \left[-E_{R} / k_{B} T_{n}\right]$, characterized by the two activation energies, $E_{D}$ and $E_{R}$ ( $k_{B}$ being the Boltzmann constant). The time evolution of the network is obtained by Monte Carlo simulations which update the network resistance after breaking and recovery processes, according to an iterative procedure described in details in Ref. ${ }^{21}$ The sequence of successive configurations provides a resistance signal, $R(t)$, after an appropriate calibration of the time scale. Then, depending on the stress conditions $\left(I\right.$ and $\left.T_{0}\right)$ and on the network parameters (size, activation energies and other parameters related to the material like $r_{0}$ and $\alpha$ ), the network either reaches a steady state or undergoes an irreversible electrical failure ${ }^{21,22}$. This latter possibility is associated with the achievement of the percolation threshold, $p_{c}$, for the fraction of broken resistors ${ }^{25}$. Therefore, for a given network at a given temperature, a threshold current value, $I_{B}$, exists above which electrical breakdown occurs $^{21}$. For values of the current below this threshold, the steady state of the network is characterized by fluctuations of the fraction of broken resistors, $\delta p$, and of the resistance, $\delta R$, around their respective average values $\langle p\rangle$ and $\langle R\rangle$. In particular, we underline that in the vanishing current limit (random percolation) ${ }^{26}$, the ratio $\left(E_{D}-E_{R}\right) / k_{B} T_{0}$ determines the average fraction of defects and thus the level of disorder inside the network. In the following we analyze the results of simulations performed by considering networks of different sizes, with different levels of disorder and stressed by different currents at room temperature, $T_{0}=300(\mathrm{~K})$. In all the cases we take $E_{D}=0.170(\mathrm{eV}), r_{0}=1(\Omega), \alpha=10^{-3}\left(\mathrm{~K}^{-1}\right)$, $A=5 \times 10^{5}(\mathrm{~K} / \mathrm{W})$ (these values are chosen as physically reasonable). The values of $N$ range between $100 \div 150$, while $E_{R}$ between $0.026 \div 0.164(\mathrm{eV})$.

\section{RESULTS}

The resistance of a $100 \times 100$ network is reported as a function of the time in Fig. 1. The different curves, starting from the bottom, are obtained for increasing values of the external current. In particular, all the evolutions in Fig. 1 are obtained by taking the activation energy of the recovery process equal to $E_{R}=0.103 \mathrm{eV}$, a 
value which leads to a network with an intermediate level of disorder. The first three curves are associated with steady states of the network. More precisely, the lowest curve corresponds to the linear response regime and it is obtained for $I=0.0013 A<I_{0}$, where $I_{0}$ is the current value associated with the onset of the nonlinearity of the I-V characteristic ${ }^{21}$. The second curve corresponds to the nonlinear regime and it is obtained for $I=0.70$ A. The third corresponds to the threshold for electrical breakdown which occurs for $I>I_{B}=0.95(A)$. By contrast, the highest curve displays the resistance evolution of a network undergoing electrical breakdown and it is obtained for a current $I=1.05(A)$. Overall, Fig. 1 illustrates qualitatively two important features of the electrical response of a conducting film. First, it evidences that the linear regime occurring for $I<I_{0}$ is followed by a nonlinear regime where the average resistance increases significantly at increasing current ${ }^{21,17}$. Second, it shows that the amplitude of the resistance fluctuations increases strongly with the external bias. This amplification of the fluctuations becomes particularly important when the current reaches the threshold for breakdown (third curve from the bottom in Fig. 1). A detailed analysis of the behavior of the average resistance and of the relative variance of resistance fluctuations as a function of the current can be found in Refs. ${ }^{21,22}$

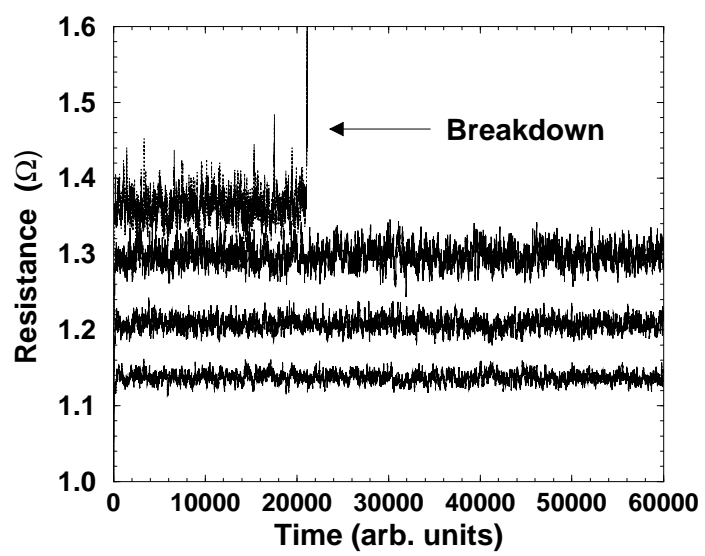

FIG. 1. Resistance evolutions of a $100 \times 100$ network stressed by increasing current values. Starting from the bottom: $I=0.0013$ A (linear regime), $I=0.70$ A (nonlinear regime), $I=0.95$ A (threshold current), $I=1.05$ A (breakdown). The recovery energy is $E_{R}=0.103 \mathrm{eV}$ and corresponds to an intermediate level of disorder.

The distributions of the resistance fluctuations, $\delta R$, for the three steady state signals in Fig. 1 are reported in Fig. 2. Precisely, by denoting with $\Phi$ the PDF of the $\delta R$ distribution and with $\sigma$ the root mean square deviation from the average resistance value, we plot in Fig. 2 on a lin-log scale the product $\sigma \Phi$ as a function of $(\langle R>-R) / \sigma$. This normalized representation, by making the distribution independent of its first and second moments, is particularly convenient to explore the functional form of any distribution ${ }^{3,4}$. Here the PDFs have been calculated by considering time series containing about $1.2 \times 10^{6}$ resistance values. In this figure, the data represented by crosses, small circles and up triangles are obtained respectively for $I=0.0013,0.70,0.95$ A (i.e. linear regime, nonlinear regime and threshold for breakdown). For comparison, in this figure we also report with a dashed curve the Gaussian distribution (which in this normalized representation has zero mean and unit variance) and, with a continuous curve, the BHP distribution ${ }^{3,4}$. By defining the normalized variable, $y \equiv(R-<R>) / \sigma$, the normalized PDF, $\Pi(y) \equiv \sigma \Phi(y)$ and $x \equiv b(y-s)$, the BHP distribution has the following functional form ${ }^{4}$ :

$$
\Pi(y)=K\left[e^{x-e^{x}}\right]^{a}
$$

where $a=\pi / 2, b=0.936 \pm 0.002, s=0.374 \pm 0.001$ and $K=2.15 \pm 0.01^{4}$. This expression can be considered as a generalization of the Gumbel distribution, which is often associated with the occurrence of rare events. Figure 2 shows a remarkable deviation from the Gaussian behavior when the network is stressed by the threshold current $I_{B}$. We have found that in this case the PDF is well fitted by the Eq. (2), once the parameters $a, b$, $s$ and $K$ are taken as fitting parameters ${ }^{27}$ In any case, we emphasize the fact that, at least for a network with intermediate level of disorder, such as that considered in Figs. 1 and 2, the deviations from Gaussianity remain weak even for current values in the nonlinear regime and become important only close to the electrical breakdown.

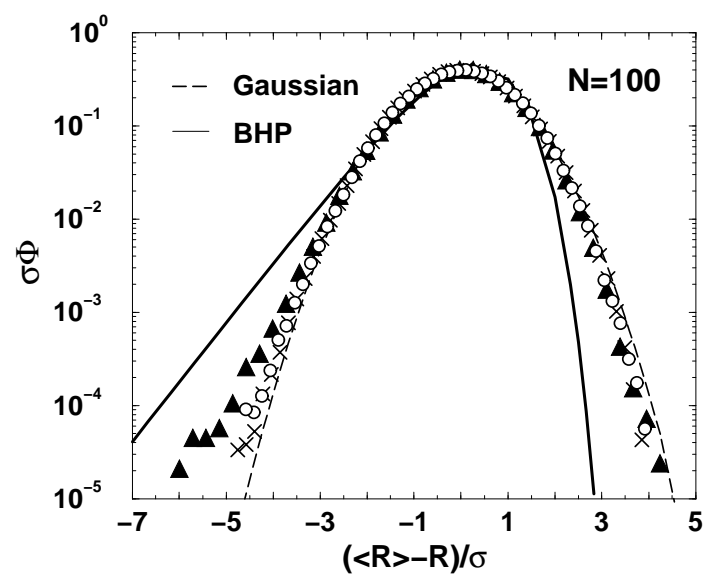

FIG. 2. Normalized PDF of resistance fluctuations for a network of size $100 \times 100$ biased by $I=0.0013$ A (crosses, linear regime), $I=0.70 \mathrm{~A}$ (small circles, nonlinear regime), $I=0.95$ A (up triangles, threshold current). The recovery energy is the same of Fig. 1. The thick solid curve and the dashed one correspond to the BHP and Gaussian distributions, respectively.

The effect of the size of the network on the distribution of resistance fluctuations, is analyzed in Figs. 3 and 4. Figure 3 compares the PDF obtained for a current in the linear regime and applied to a network $100 \times 100$ (the data, reported by crosses, are the same of Fig. 2) with the PDF obtained for a current in the same regime 
applied to a network $125 \times 125$ (diamonds). The two networks have the same parameters and differ only for the size, thus they share the same level of internal disorder. Figure 3 shows that in the linear regime the distribution of $\delta R$ is Gaussian for all system sizes. In case, a weak non-Gaussian tail appears for very small systems that vanishes for systems of larger size.

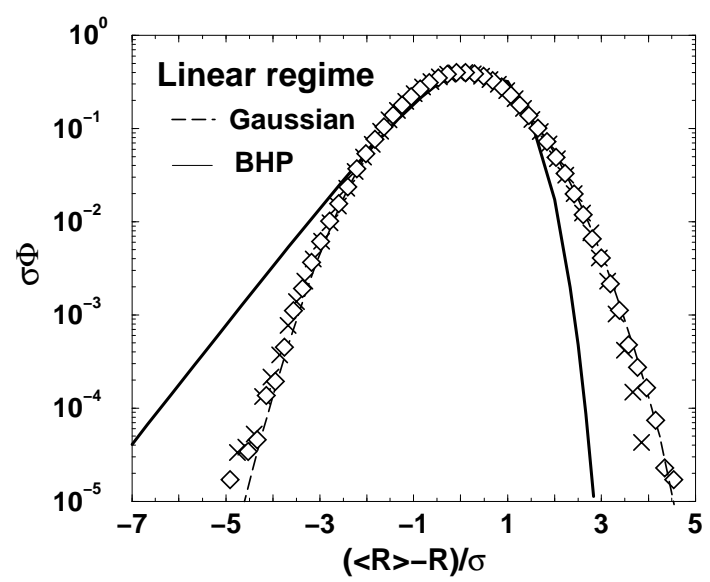

FIG. 3. Normalized PDF of resistance fluctuations for networks of size $100 \times 100$ (crosses) and $125 \times 125$ (diamonds). The networks are biased by currents in the linear regime. The recovery energy is the same of Fig. 1. The thick solid curve and the dashed one correspond to the BHP and Gaussian distributions, respectively.

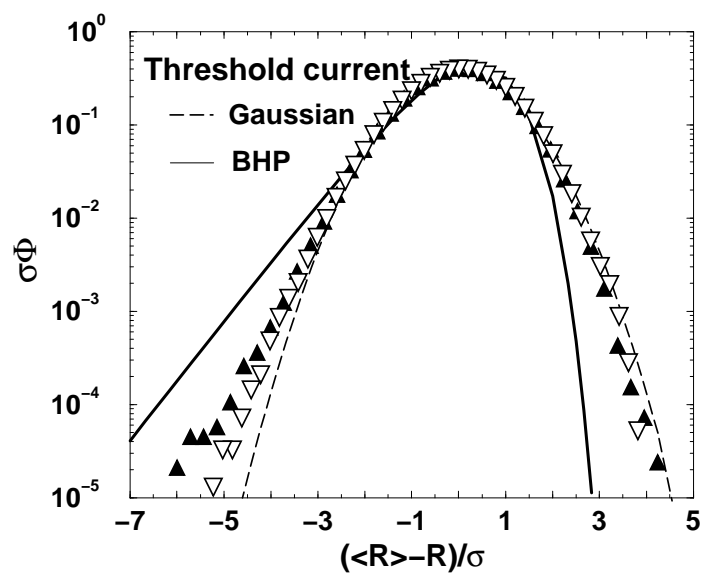

FIG. 4. Normalized PDF of resistance fluctuations for networks of size $100 \times 100$ (up triangles) and $125 \times 125$ (down triangles). The networks are biased by $I=0.95 \mathrm{~A}$ and $I=1.15$ A which correspond to their respective threshold for breakdown. The recovery energy $E_{R}$ is the same of the previous figures and the thick solid and the dashed curves have the same meaning of Fig. 3 .

Figure 4 displays a comparison similar to that in Fig. 3 for two networks of size $100 \times 100$ (up triangles, same data of Fig. 2) and $125 \times 125$ (down triangles), stressed by currents corresponding to the threshold for breakdown. The current values are $I=0.95,1.15 \mathrm{~A}$, respectively for the networks with $N=100$ and $N=125$. We can see that in both cases the PDFs exhibit non-Gaussian tails. However, the same figure shows that the non-Gaussianity is weaker for the system of larger size. By performing a detailed investigation on systems of different sizes we have found that this trend is systematic ${ }^{28}$. Therefore, we conclude that, at least when networks with intermediate level of disorder are considered, as in the case of Figs. 1-4, the deviations from Gaussianity are related to the finite size of the system.

Now, before discussing the role of disorder in the breakdown process, it is convenient to distinguish between the intrinsic disorder and the disorder driven by the external bias. For given values of $T_{0}$ and $E_{D}$, the average fraction of broken resistors in the vanishing current limit, $\langle p\rangle_{0}$, is only determined by the recovery energy $E_{R}{ }^{26}$. This average fraction of defects represents an intrinsic property of the system, which characterizes the conducting material and sets the level of disorder inside the network in absence of an external bias. We note nate the steady state of a network in this vanishing current limit is determined by the competition between two random percolations ${ }^{26}$. On the other hand, for $I>I_{0}$, there is also an additional component of disorder due to the defects generated by the external current ${ }^{21}$. Therefore, the average fraction of defects $\langle p\rangle$ becomes dependent on the current and its relative variation, $\left.[<p\rangle-<p\rangle_{0}\right] /<p>_{0}$, scales $\operatorname{as}^{21}\left(I / I_{0}\right)^{2}$. Of course, this dependence of $\langle p\rangle$ on $I$ is important because it actually drives the system towards the breakdown. However, it is also crucial to point out the role of the intrinsic disorder on the breakdown process and its effect on the distribution of resistance fluctuations. Therefore, in the following we will consider networks with different values of the recovery activation energy $E_{R}$.

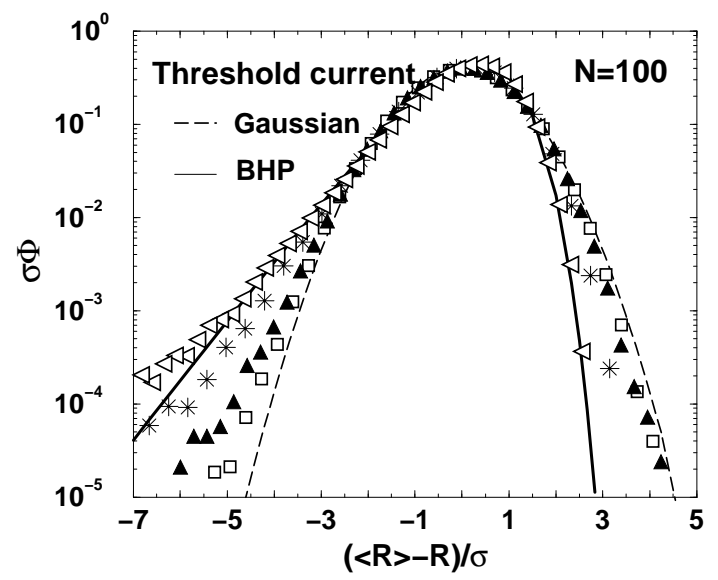

FIG. 5. Normalized PDF of resistance fluctuations of $100 \times 100$ networks at increasing $E_{R}$ values (level of disorder). In all the cases the current value corresponds to the respective threshold for breakdown. Precisely, squares: $E_{R}=0.026, I_{B}=3.5$; up triangles: $E_{R}=0.103, I_{B}=0.95$; stars: $E_{R}=0.155, I_{B}=0.11$, left triangles: $E_{R}=0.164$, $I_{B}=0.009$ (energies in $\mathrm{eV}$, currents in A). 
In a previous work ${ }^{29}$ we have shown that for an arbitrary value of $E_{R}$ satisfing the stability condition ${ }^{26,22}$, i.e. providing a steady state of the network, the electrical breakdown is associated with a first order phase transition. This result agrees with the behavior observed in electrical breakdown experiments, performed in the Joule regime of composites ${ }^{17,18}$. Nevertheless, it can be shown ${ }^{30}$ that when $E_{R}$ reaches its maximum value compatible with a steady state, $E_{R, M A X}$, the conductorinsulator transition becomes of the second order. This change in the order of the transition, when going from low to high disordered systems, has been also predicted by Andersen et al. ${ }^{14}$

Therefore, we report in Fig. 5 the PDFs of resistance fluctuations of $100 \times 100$ networks characterized by different values of $E_{R}$. The PDFs have been calculated for current values corresponding to the breakdown threshold. We can see that the non-Gaussianity of the distribution at $I=I_{B}$ increases systematically at increasing values of $E_{R}$ (i.e. at increasing level of the intrinsic disorder). In particular, Fig. 5 shows that when the value of $E_{R}$ is very close to $E_{R, M A X}$, and thus the system approaches the critical point, the PDF achieves (left triangles) the BHP form. In these conditions, the PDF should become independent of the system size ${ }^{3,4}$.

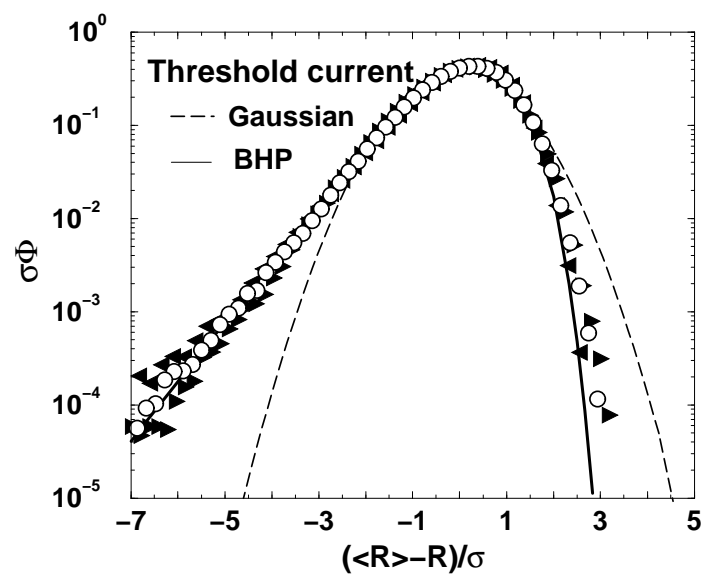

FIG. 6. Normalized PDF of resistance fluctuations for networks of increasing size and close to the critical conditions: the recovery energy is $E_{R}=0.164 \mathrm{eV}$ and the bias current corresponds to the threshold for breakdown. Precisely, left triangles refer to a network $100 \times 100$ biased by $I=0.0090$ A, circles to a network $125 \times 125$ biased by $I=0.0011 \mathrm{~A}$ and right triangles to a network $150 \times 150$ and $I=0.0013 \mathrm{~A}$.

Indeed, Fig. 6 confirms this behavior. In this figure we report the PDFs calculated for the three networks of size $100 \times 100$ (left triangles, same data of Fig. 5), $125 \times 125$ (circles) and $150 \times 150$ (right triangles). In all the cases the recovery energy is $E_{R}=0.164 \mathrm{eV} \approx E_{R, M A X}$ and the external current corresponds to the threshold value for breakdown $\left(I_{B}=0.0090,0.011,0.013 \mathrm{~A}\right.$, respectively). The data in Fig. 6 shows that, when $E_{R}$ is very close to $E_{R, M A X}$ and thus the system approaches the critical point, the PDFs of resistance fluctuations become independent of the system size and they are well described by the BHP distribution.

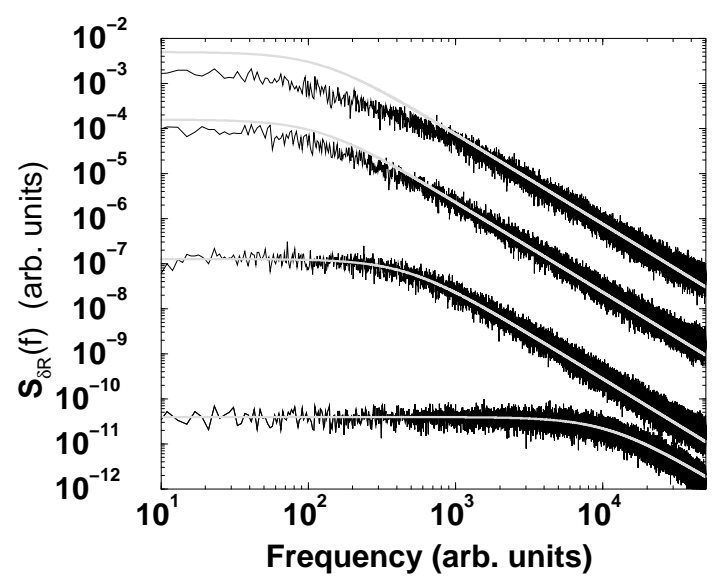

FIG. 7. Power spectral density of resistance fluctuations of $125 \times 125$ networks for increasing $E_{R}$ values. Precisely, starting from the bottom: $E_{R}=0.026,0.103,0.155,0.164 \mathrm{eV}$. In all cases the current corresponds to the respective threshold for breakdown (respectively: $I=4.4,1.15,0.14,0.011$ A). For visual reasons the lowest spectrum has been shifted down by multipling it by a factor $5 \times 10^{-3}$. The gray lines represent the best-fit with a Lorentzian spectral density.

Other interesting information about the system can be extracted from the spectral analysis of resistance fluctuations ${ }^{2,6,9}$. Hence we report in Fig. 7 the power spectral density of resistance fluctuations of $125 \times 125$ networks at increasing values of $E_{R}$. All the spectra are calculated for networks stressed by currents corresponding to the threshold for electrical breakdown. Starting from the bottom, the curves are obtained respectively for $E_{R}=0.026,0.103,0.155$ and $0.164 \mathrm{eV}$ (the same values of $E_{R}$ considered in Fig. 5). Basically all the spectra display a Lorentzian behavior which witnesses an exponential decay of the auto-correlation function of resistance fluctuations $^{2}$ (the gray curves in this figure represent the best-fit with a Lorentzian distribution). Moreover, we can see that for increasing values of $E_{R}$, i.e. for increasing level of intrinsic disorder, the corner frequency of the spectra is systematically shifted towards lower values. This occurs because, at increasing values of $\langle p\rangle$, the network approaches the percolation threshold, thus the correlation length increases as $\xi \sim\left|\langle p\rangle-p_{c}\right|^{-\nu}$ (where $\nu$ is the correlation length exponent) ${ }^{25}$ and the same occurs for the correlation time of resistance fluctuations. However, we note that for the two upper spectra in Fig. 7 the fit with a Lorentzian curve appears progressively less satisfactory in the low frequency region. On the other hand, these spectra are obtained by taking the value of $E_{R}$ near or very near to $E_{R, M A X}$ and thus correspond to networks progressively closer to the critical conditions. This anomalous behavior in the low frequency region of the power specral density of resistance fluctuations in the case of networks close to criticality, could 
even suggest the emergence of a $1 / f$ behavior. Longer time series are required to point out the behavior of the spectra in this frequency region.

\section{CONCLUSIONS}

We have studied the distribution of the resistance fluctuations of conducting thin films with different levels of internal disorder. The study has been performed by describing the film as a resistor network in a steady state determined by the competition of two biased stochastic processes, according to the SBRN model ${ }^{20-22}$. We have considered systems of different sizes and under different stress conditions, from the linear response regime up to the threshold for electrical breakdown. A remarkable non-Gaussianity of the fluctuation distribution is found near breakdown. This non-Gaussianity becomes more evident at increasing the degree of disorder of the network. As a general trend, these deviations from Gaussianity are related to the finite size of the system and they are found to vanish in the large size limit. However, near the critical point of the conductor-insulator transition, the non-Gaussianity is found to persist in the large size limit and is well fitted by the universal Bramwell-HoldsworthPinton distribution ${ }^{3,4}$. Furthermore, in the last case, the non-Gaussianity seems to be associated with an anomalous behavior in the low frequency region of the specral density of resistance fluctuations.

\section{ACKNOWLEDGEMENTS}

Partial support from the cofin-03 project "Modelli e misure di rumore in nanostrutture" financed by Italian MIUR and from the SPOT-NOSED project IST-200138899 of EC is gratefully acknowledged.

${ }^{1}$ B.K. Chakrabarti and L. Benguigui, Statistical Physics of Fracture and Breakdown in Disordered Systems, Oxford Univ. Press, Oxford (1997).

${ }^{2}$ M. B. Weissman, Rev. Mod. Phys., 60, 537 (1988).

${ }^{3}$ S.T. Bramwell and P.C.W. Holdsworth and J. F. Pinton, Nature, 396, 552 (1998).

${ }^{4}$ S.T. Bramwell, K. Christensen, J. Y. Fortin, P. C. W. Holdsworth, H.J. Jensen, S. Lise, J. M. López, M. Nicodemi, J. F. Pinton and M. Sellitto, Phys. Rev. Lett., 84, 3744 (2000).
${ }^{5}$ V. Aji and N. Goldenfeld, Phys. Rev. Lett., 86, 1107 (2001).

${ }^{6}$ T. Antal, M. Droz, G. Györgyi, Z. Rácz, Phys. Rev. E, 65, 046140 (2002).

${ }^{7}$ V. Eisler, Z. Rácz and F. Van Wijland, Phys. Rev. E, 67, 56129 (2003).

${ }^{8}$ S.T. Bramwell, J. Y. Fortin, P. C. W. Holdsworth, S. Peysson, J. F. Pinton, B. Portelli and M. Sellitto, Phys. Rev. E, 63, 041106 (2001).

9 T. Antal, M. Droz, G. Györgyi and Z. Rácz, Phys. Rev. Lett., 87, 24061 (2001).

${ }^{10}$ B. Portelli, P. C. W. Holdsworth, M. Sellitto and S.T. Bramwell, Phys. Rev. E, 64, 036111 (2001).

${ }^{11}$ K. Dahlstedt and H.J. Jensen, J. Phys. A, 34, 11193 (2001).

12 N. Vandewalle, M. Ausloos, M. Houssa, P. W. Mertens and M. M. Heyns, Appl. Phys. Lett., 74, 1579 (1999).

13 A. Hansen, S. Roux and E. L. Hinrichsen, Europhys. Lett., 13, 341 (1990).

${ }^{14}$ L. Lamaignère, F. Carmona and D. Sornette, Phys. Rev. Lett., 77 (1996).

15 J. V. Andersen, D. Sornette and K. Leung, Phys. Rev. Lett., 782140 (1997).

${ }^{16}$ S. Zapperi, P. Ray, H. E. Stanley and A. Vespignani, Phys. Rev. Lett., 78, 1408 (1997).

${ }^{17}$ C. D. Mukherjee, K. K. Bardhan and M. B. Heaney, Phys. Rev. Lett., 83, 1215 (1999).

${ }^{18}$ C. D. Mukherjee and K. K. Bardhan, Phys. Rev. Lett., 91, 025702-1 (2003).

${ }^{19}$ C. Pennetta, L. Reggiani and G. Trefan, Phys. Rev. Lett., 84, 5006 (2000).

${ }^{20}$ C. Pennetta, G. Trefan and L. Reggiani, in Unsolved Problems of Noise and Fluctuations, Ed. D. Abbott, L. B. Kish, AIP Conf. Proc., 551, 447 (1999).

${ }^{21}$ C. Pennetta, L. Reggiani, G. Trefan and E. Alfinito, Phys. Rev. E, 65, 066119 (2002).

${ }^{22}$ C. Pennetta, Fluct. Noise Lett., 2, R29 (2002).

${ }^{23}$ C. Pennetta, L. Reggiani, G. Trefan, F. Fantini, A. Scorzoni and I. De Munari, J. Phys. D: Appl. Phys., 34, 1421 (2001).

${ }^{24}$ Z. Gingl, C. Pennetta, L. B. Kish and L. Reggiani, Semic. Sci. Techn., 11, 1770 (1996).

${ }^{25}$ D. Stauffer and A. Aharony, Introduction to Percolation Theory, (Taylor and Francis, 1992).

${ }^{26}$ C. Pennetta, G. Trefan and L. Reggiani, Phys. Rev. Lett., 85, 5238 (2000).

${ }^{27}$ C. Pennetta, E. Alfinito, L. Reggiani and S. Ruffo, Semic. Sci. Techn., 19, S164 (2004)

${ }^{28}$ C. Pennetta, E. Alfinito, L. Reggiani and S. Ruffo, Physica A, in print (2004).

${ }^{29}$ C. Pennetta, E. Alfinito and L. Reggiani, in Unsolved Problems of Noise and Fluctuations, Ed. S. M. Bezrukov, AIP Conf. Proc., 665, 480 (2004).

${ }^{30}$ C. Pennetta and E. Alfinito, unpublished. 